\title{
Anterior Segment Ischaemia
}

\author{
J. P. LEE and J. M. OLVER \\ London
}

Anterior Segment Ischaemia or Anterior Segment Necrosis (ASI), (Figure 1) was first described by Schmidt ${ }^{1}$ in 1874 and Hayreh ${ }^{2}$ states that it has occurred in association with aortic arch syndrome, carotico-cavernous fistula, ${ }^{3}$ acute angle closure glaucoma, irradiation, herpes zoster ophthalmicus, rubella, retinal detachment, sickle cell disease and a variety of other conditions. It may also occur spontaneously in high myopes; but the vast majority of cases follow either strabismus surgery with detachment of rectus muscles or retinal detachment surgery. Anterior segment ischaemia following experimental transposition in monkeys was described but not recognised by Leinfelder and Black in $1941,{ }^{4}$ and was first described after three muscle transposition surgery in humans by Stucchi and Bianci in 1957. ${ }^{5}$ Anterior segment ischaemia after retinal detachment surgery was first described by Wilson and Irvine in $1955^{\circ}$ following encirclement and has since been described following encirclement and scleral buckling procedure with and without rectus muscle detachment ${ }^{7}$ and following scleral buckling alone. ${ }^{8}$ Hayreh $^{9}$ has also produced the condition experimentally by occlusion of the vortex veins.

The symptoms and signs of ASI syndrome are generally well known, although happily, most ophthalmologists have not induced the condition. It is worth noting that the clinical syndrome is slightly dissimilar following strabismus surgery and retinal detachment surgery, clearly reflecting the different pathogenesis. With both aetiologies the patient complains of pain, photophobia and blurred vision initially, often at the first dressing, or a day or so later. Following squint surgery the signs include conjunctival oedema, deep anterior chamber, flare and cells, pupillary distortion and dilation, lens opacities and hypotony with later iris atrophy. The commonest long-term symptom is photophobia secondary to pupil changes and iris atrophy, which tends to persist. The syndrome after detachment surgery, although similar, usually has shallowing of the anterior chamber, marked flare, the intra-ocular pressure is more commonly raised rather than lowered and the eye is generally more congested.

How common is anterior segment ischaemia? Exact figures for this are hard to obtain and rely on retrospective case analysis and ques-

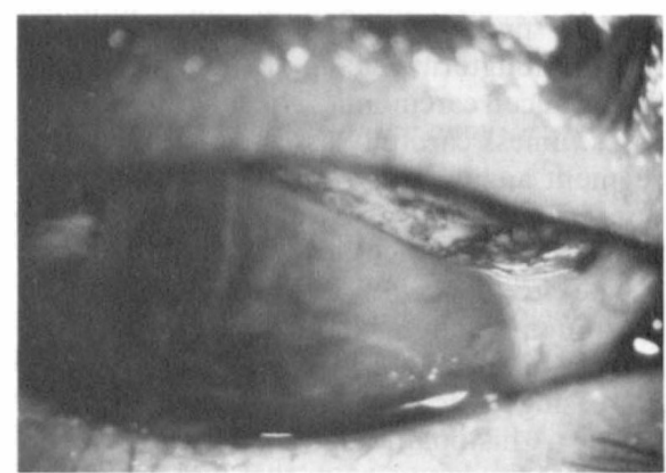

Fig. 1. Right eye. Acute anterior segment ischaemia on the first post-operative day following superior and inferior rectus recessions, showing chemosis, striate keratitis and irregular dilated pupil. 
tionnaires. Simon et al. ${ }^{10}$ in 1984 reported a large series of cases of ASI following strabismus surgery. The study was published in $\mathrm{JPed}$ Ophthalmol \& Strabismus with a discussion by France, and clearly led to discussion of prevalence, because in 1986 France and Simon ${ }^{11}$ reported the findings of a postal questionnaire study sent to all members of the American Association for Pediatric Ophthalmology and Strabismus (AAPOS) regarding ASI. Questions asked included the type of operations resulting in ASI, medical history, age of patient and the reported incidence per strabismus operation was estimated at $1: 13,333$. This figure has been widely reported but it must be remembered that these were cases of severe ASI (Grade 4, Infarction by clinical and fluorescein grading $^{12}$ ) which had significant signs and symptoms. Also, this figure represents all strabismus operations, horizontal and vertical, children and adults. We reported preliminary results on a prospective series, ${ }^{12}$ using iris angiography, of adult strabismus surgery in which signs of anterior segment ischaemia were detectable (pupil changes and/or mild uveitic signs) but not severe in six out of 35 adult cases (all types of surgery), although no case showed the full blown disease. This study has since been expanded and it is of note that out of 41 patients undergoing primary vertical rectus muscle surgery four patients developed symptomatic Grade 3 ASI with uveitis between three and seven days following two muscle surgery; in one of these patients marked pupil distortion and photophobia resulted longterm. That patient has since died from oat cell carcinoma. The majority of such cases, unless carefully assessed with anterior segment angiography, will be labelled 'postoperative' uveitis, and will usually spontaneously improve.

The frequency of ASI following scleral buckling procedures has been reported clinically as high as $3 \%$ in non-sickle cell patients increasing to $71 \%$ in sickle cell haemaglobinopathy. ${ }^{7}$ A histopathological study ${ }^{13}$ of 49 eyes obtained at post mortem that had previously had retinal detachment surgery found that four eyes all within 16 days post-surgery (8.2\% of total) showed evidence of ASI with ciliary epithelial necrosis, but there was no available correlation with clinical signs.

\section{What kind of surgery causes ASI?}

The traditional received wisdom is that two rectus muscles can be safely detached in one procedure, but that three or more are dangerous. The AAPO\&S study supports this view, with transpositions and Jensen procedures having the highest rate of ASI, although some two muscle procedures also caused the problem. The sole case of ASI that one of us (JPL) has had was in a woman of 40 years (see Figure 1) who had surgery on both vertical recti of one eye twenty years after horizontal rectus surgery, but in whom re-exploration of the deep episcleral vasculature may have disturbed important collaterals and precipitated sector infarction. Fells and Marsh $^{14}$ also described ASI after two rectus muscle surgery.

Hayreh and $\mathrm{Scott}^{15}$ have demonstrated that vertical rectus muscle surgery produces corresponding segmental delay in adult patients immediately post-operatively. In our clinical study $^{12}$ we identified not only that primary vertical rectus muscle surgery resulted in reproducible sector-delay but that secondary vertical rectus muscle surgery (subsequent surgery on the same muscle) did not. This observation, together with episcleral angiographic findings of the strabismus surgical scar led us to appreciate that following initial surgery, further surgery on the same muscle was unlikely to cause further delay since restoration of blood flow was largely by remodelling of the collateral circulation and not recanalisation of the anterior ciliary arteries. This has since been confirmed in an experimental squint model using vascular casting. ${ }^{16}$

The vascular anatomy of the anterior segment is an important consideration in strabismus surgery. Vertical recti carry a greater contribution relative to the anterior segment via the anterior ciliary arteries in man, whilst the medial horizontal anterior ciliary arteries are supplemented by the medial long posterior ciliary artery supply and the lateral anterior ciliary artery does not itself normally contribute to the uveal circulation.

Clearly three rectus muscle surgery will involve either one or two vertical recti, and therefore an increased risk of anterior segment ischaemia. Since the lateral rectus ACA makes no direct contribution to the anterior 
segment, a combination of superior, inferior and medial rectus is likely to be the most potentially disastroussurgery. This, of course, is the combination one would wish to use in surgery for unrecovered sixth nerve palsy, but such procedures are the most hazardous. Transposition alone carries an additional risk of developing ASI if the lateral perforators are damaged, since remodelling following squint surgery is located partly in the episcleral collaterals.

It is less easy to be sure what kind of surgery causes ASI after retinal detachment, but it is clearly multifactorial and may involve a combination of procedures; long posterior ciliary artery occlusion and vortex vein occlusion following encirclement or scleral buckles with anterior ciliary arteries either being damaged directly by detachment of a rectus muscle (rarely practiced now) or indirectly during tractional manipulations. Chignell and Easty ${ }^{17}$ described iris angiographic changes following retinal detachment surgery in the absence of symptomatic ischaemia. Hayreh ${ }^{18}$ has suggested that the common factor in all responsible detachment surgery is engorgement of the venous compartment of the anterior segment by interference with posterior drainage of venous blood to the vortex veins.

A number of case reports have emphasised the role of other predisposing factors in ASI. Otherwise 'safe' surgery has precipitated the condition in patients with carotid insufficiency, ${ }^{19}$ chronic lymphatic leukaemia ${ }^{20}$ and other hyperviscosity states.

\section{What is the mechanism of ASI?}

The presumed sequence of events is hypoperfusion $>$ tissue hypoxia $>$ cell death $>$ inflammatory response. Clearly this sequence may be interrupted at any point, and may not progress all the way. Similarly, the potential for manipulating each stage medically also exists.

In squint surgery, interruption of the anterior ciliary arteries (ACA) may be accompanied by other disruption of the deep episcleral collateral vessels (anterior episcleral arterial circle and scleral perforators) between the ACAs either by cautery or surgical trauma.

In detachment surgery, although the ACAs are obstensibly spared, large explants and over liberal cautery may cause insufficiency. In addition, there may be interference with the long posterior ciliary arteries (LPCAs) by encirclement or plombs. Venous engorgement, as suggested by Hayreh, may be caused by the same means.

Hypoperfusion is clearly a pre-existing factor in carotid insufficiency, carotico-cavernous fistula and hyperviscosity states.

\section{Experimental studies of the anterior ocular circulation}

\section{(a) Relative contributions of ACAs and LPCAs}

Wilcox and Keough in a series of experiments $^{21-23}$ between 1980 and 1983 involving perfusion of labelled microspheres in macaque monkeys following various patterns of selective rectus tenotomy concluded that the ACAs had a greater importance than the LPCAs. Virdi and Hayreh ${ }^{24}$ in 1987 conducted a large experiment on a series of primate eyes using angiography and examination after a variety of rectus muscle disinsertions. Severe ASI followed detachment of all four recti, whereas occlusion of the LPCAs produced no detectable effect. They also pointed out that secondary or staged surgery had a protective effect, that, when all four recti were removed in two stages, no ASI occurred, suggesting some remodelling of the circulation. In both these studies it is worth noting that the experimental subjects were relatively 'youthful' and an experimental study on elderly primate eyes has yet to be undertaken.

\section{(b) Anatomical studies of the anterior segment vasculature}

(i) Vascular casting studies

One of the advantages of scanning electron microscopy of vascular casts is that arteries and veins can be clearly differentiated by their characteristic endothelial nuclear impressions. Morrison and Van Buskirk elegantly demonstrated a three tiered collateral supply of the anterior segment in monkeys, ${ }^{25}$ from sequential micro-dissection of corrosion casts. These consisted of an anterior episcleral arterial circle, intra-muscular arterial circle and major arterial circle. In $\operatorname{man}^{26}$ a similar but not identical morphology exists. The 
intra-muscular circle is not a complete circle; intra-muscular arteries arise as branches of the long posterior ciliary arteries from which anterior communicating arteries supply the major arterial circle. Perforating branches of the medial anterior ciliary arteries connect to the intra-muscular arteries. The intra-muscular arteries travel progressively anteriorly towards the limbus to merge with the major arterial circle. However, the major arterial circle vertically is supplied directly by perforating branches of the vertical anterior ciliary arteries. The major arterial circle is composed of overlapping circumferential segments and is also not a complete circle. The anterior episcleral arterial circle exists also in man, formed by the anterior ciliary arteries. The lateral anterior ciliary artery however does not directly connect with the deep collateral circulation. . $^{25,26,27}$

\section{(ii) Fluorescein angiographic studies}

Low dose fluorescein angiography enables us to study the episcleral arterial collaterals in man in detail. ${ }^{28}$ Our own observations confirm the findings by several other authors ${ }^{29-32}$ that direction of blood flow in the anterior ciliary artery scleral perforators is frequently from within the eye outwards. However, the clinical, experimental and anatomical evidence in man demonstrates functionally that vertical and medial anterior ciliary arteries are normal contributors to the anterior uveal blood supply. This apparent paradox may be explained by differing flow dynamics in agonist and antagonist muscles and the metabolic requirements of the ciliary body.

\section{Practical Advice \\ What is safe to do in strabismus surgery?}

In general, for primary rectus muscle surgery, two horizontal recti are always safe, unless a predisposing factor exists. Three muscles including one vertical rectus is also safe under the same conditions as long as the anterior episcleral arterial circle is treated with care.

Two vertical recti or three muscles including two vertical recti are much more likely to cause ASI as the vertical recti, as already observed, ${ }^{12,18}$ make a large contribution to the anterior ciliary circulation and anterior uveal circulation.
$\mathrm{We}^{12}$ and Virdi and Hayreh ${ }^{24}$ have shown clearly the effect of staged surgery, that is to say subsequent detachment of different rectus muscles after initial rectus muscle surgery (our use of the term secondary surgery is restricted to second surgery on the same muscle and not used for staged surgery). Although we could expect a cumulative effect on the anterior segment circulation, in fact we find that three or four recti may be detached safely as long as this occurs in two or more surgical procedures. Compensatory mechanisms including increased flow in the LPCAs in the acute post-operative stage and long term remodelling of the collateral circulation is the most probable explanation.

Are there surgical techniques to avoid ASI? The simplest advice is to operate with care, identifying the ACAs and the collaterals to the AEAC. Dissection should be careful and cautery used sparingly. Muscle-splitting techniques such as the Jensen procedure may be employed, although these probably have a fairly profound effect on anterior segment perfusion and cases of ASI following these procedures have been reported. ${ }^{33}$

McKeown et al. ${ }^{34}$ have recently revived interest in the technique of anterior ciliary vessel preservation during strabismus surgery. However their paper largely describes the method without much data on the state of the anterior segment circulation.

For unrecovered sixth nerve palsy, we have recently reported ${ }^{35}$ the use of Botulinum Neurotoxin A as a chemodenervation of the medial rectus prior to lateral transposition of the vertical recti, giving effective abduction with two-muscle surgery. Recently, de Smet et al. ${ }^{36}$ have reported one case of successful treatment of ASI with hyperbaric oxygen, which appears rational in severe instances.

Techniques in detachment surgery should probably include careful surgical technique, especially round the area of the LPCAs and vortex veins, and gentle encirclement.

\section{Conclusions}

We have discussed the condition of anterior segment ischaemia (ASI), its causation, pathophysiology and the rationale of avoiding it. It is hoped that newer techniques of inves- 
tigation and treatment will further reduce the incidence of this potentially disastrous condition.

\section{References}

${ }^{1}$ Crock G: Clinical syndromes of anterior segment ischaemia. Trans Ophthalmol Soc UK 1967, 87: 513-32.

${ }^{2}$ Hayreh SS: Ischaemia of the anterior segment of the eye. Irish Faculty of Ophthalmology Yearbook 1979/1980, 21-31.

${ }^{3}$ Sanders MD and Hoyt WF: Hypoxic ocular sequelae of carotid cavernous fistulae. $\mathrm{Br} \mathrm{J}$ Ophthalmol 1969, 53: 82-97.

${ }^{4}$ Leinfelder PJ and Black NM: Experimental transposition of the extra-ocular muscles in monkeys. Preliminary report. Am J Ophthalmol 1941, 24: 1115-20.

${ }^{5}$ Stucchi $\mathrm{C}$ and Bianchi G: Depigmentation en secteur de l'iris consecutive a des transplantations musculaires. Ophthalmologica 1957, 133: 231-6.

${ }^{6}$ Wilson WA and Irvine SR: Pathologic changes following disruption of blood supply to iris and ciliary body. Trans Am Acad Ophthalmol Otolaryng 1955, 59: 501-2.

${ }^{7}$ Ryan SJ and Goldberg MF: Anterior segment ischaemia following scleral buckling in sickle cell hemoglobinopathy. Am J Ophthalmol 1971, 72: 35-50.

${ }^{8}$ Robertson DM: Anterior segment ischaemia after segmental episcleral buckling and cryopexy. Am J Ophthalmol 1975, 79: 871-4.

${ }^{9}$ Hayreh SS and Baines JAB: Occlusion of the vortex veins. An experimental study. Br J Ophthalmol 1973, 57: 217-38.

${ }^{10}$ Simon JW, Price EC, Krohel GB, Poulin RW, Reinecke RD: Anterior segment ischaemia following strabismus surgery. J Paed Ophthalmol Strabis 1984, 21: 179-84. Discussion by France TD $\mathrm{p} 185$.

${ }^{11}$ France TD and Simon JW: Anterior segment ischaemia syndrome following muscle surgery: The AAPO\&S experience. J Paed Ophthalmol Strabis 1986, 23: 87-91.

${ }^{12}$ Olver JM and Lee JP: The effects of strabismus surgery on anterior segment circulation. Eye 1989, 3: 318-26.

${ }^{13}$ Wilson DJ and Green WR: Histopathologic study of the effect of retinal detachment surgery on 49 eyes obtained post mortem. Am J Ophthalmol 1987, 103: 167-79.

${ }^{14}$ Fells P and Marsh RJ: Anterior segment ischaemia following surgery on two rectus muscles. In Reinecke R (Ed): Strabismus. New York, Grune \& Stratton, 1978, pp. 375-80.

${ }^{15}$ Hayreh SS and Scott WE: Fluorescein iris angiography. II. Disturbances in iris circulation following strabismus operation on the various recti. Arch Ophthalmol 1978, 96: 1390-400.

${ }^{16}$ Olver JM, McCartney ACE, Lee JP: The strabismic scar; a microvascular corrosion cast study. Invest Ophthalmol \& Vis Sci 1989, 30: (Suppl) 377.

${ }^{17}$ Chignell AH and Easty DL: Iris fluorescein pho- tography following retinal detachment and in certain ocular ischaemic disorders. Trans Ophthalmol Soc UK 1971, 91: 243-57.

${ }^{18}$ Hayreh SS and Scott WE: Anterior segment ischaemia following retinal detachment surgery. Mod Probl Ophthalmol 1979, 20: 148-53.

${ }^{19}$ Fells P: Vertical rectus muscle transposition to restore abduction. In: Orthoptics, Proc 2nd Int Orthop congr Ed; J Mein, JJM Bierlaagh, TEA Brummelkamp-Dons. Excerpta Medica, Amsterdam, p 299.

${ }^{20}$ Jacobs DS, Vastine DW, Urust MJ: Anterior segment ischaemia and sector iris atrophy: after strabismus surgery in a patient with chronic lymphocytic leukaemia. Ophthalmic Surg 1976, 7: 42-8.

${ }^{21}$ Wilcox LM, Keough EM, Connolly RJ, Hotte CE: The contribution of blood flow by the anterior ciliary arteries to the anterior segment in the primate eye. Exp Eye Res 1980, 30: 167-74.

${ }^{22}$ Wilcox LM, Keough EM, Connolly RJ: Regional ischaemia and compensatory vascular dynamics following selective tenotomy in primates.

${ }^{23}$ Keough EM, Connolly RJ, Ramberg-Laskaris K, Wilcox LM: Primate ocular blood flow following contiguous recti tenotomy. Ophthalmic Res 1983 , 15: 204-7.

${ }^{24}$ Virdi PS and Hayreh SS: Anterior segment ischaemia after recession of various recti. An experimental study. Ophthalmology 1987, 94: 1258-71.

${ }^{25}$ Morrison JC and Van Buskirk EM: Anterior collateral circulation in the primate eye. Ophthalmology 1983, 90: 707-15.

${ }^{26}$ Olver JM, McCartney ACE, Lee JP: Morphology of the anterior segment vasculature in man; a microcorrosion cast study. (In preparation).

${ }^{27}$ Woodlief NF: Initial observations on the ocular microcirculation in man. I. The anterior segment and extraocular muscles. Arch Ophthalmol 1980, 98: $1268-72$.

${ }^{28}$ Meyer PAR and Watson PG: Low dose fluorescein angiography of the conjunctiva and episclera. $\mathrm{BrJ}$ Ophthalmol 1987, 71: 2-10.

${ }^{29}$ Bron AJ and Easty DL: Fluorescein angiography of the globe and anterior segment. Trans Ophthalmol Soc UK 1970, 90: 339-67.

${ }^{30}$ Ikegami M: Fluorescein angiography of the anterior ocular segment. Part 1 . Hemodynamics in the anterior ciliary vessels. Acta Soc Ophthalmol Jpn 1974, 78: 39-53

${ }^{31}$ Talusan ED and Schwartz B: Fluorescein angiography; demonstration of flow pattern of anterior ciliary arteries. Arch Ophthalmol 1981, 99: 1074-80.

${ }^{32}$ Meyer PAR: Patterns of blood flow in episcleral vessels studied by low-dose fluorescein videoangiography. Eye 1988, 2: 533-46.

${ }^{33}$ Von Noorden GK: Anterior segment ischaemia following Jensen procedure. Arch Ophthalmol 1976, 94: $845-7$

${ }^{34}$ McKeown CA, Lambert HM, Usaf MC, Shore JW: Preservation of the anterior ciliary vessels during extraocular muscle surgery. Ophthalmology 1989 , 96: 499-507.

${ }^{35}$ Fitzsimmons R, Lee JP, Elston, JS: Treatment of 
sixth nerve palsy in adults with combined toxin chemodenervation and surgery. Ophthalmology 1988, 95: 1535-42.
${ }^{36}$ de Smet MD, Carruthers J, Lepawsky M: Anterior segment ischaemia treated with hyperbaric oxygen. Can J Ophthalmol 1987, 22: 381-3. 